\title{
DEFORMATION OF OPEN-CELL ALUMINUM FOAM
}

\author{
C. SAN MARCHI + and A. MORTENSEN $\dagger$ \\ Laboratory of Mechanical Metallurgy, Swiss Federal Institute of Technology in Lausanne (Ecole \\ Polytechnique Fédérale de Lausanne, EPFL), CH-1015 Lausanne, Switzerland
}

( Received 11 April 2001; received in revised form 31 July 2001; accepted 31 July 2001)

\begin{abstract}
The mechanical properties of high-purity aluminum foams produced by replication from salt precursors are measured in compression. These foams have homogeneous open-porosity, cell sizes equivalent to the particle size of the precursor salt $(\sim 500 \mu \mathrm{m}$ in this case) and relative densities near $25 \%$. Deformation is uniform and strain hardening similar to the bulk material is observed without a plateau stress. A simple analytical model based on beam theory is employed to describe the flow stress and the change in stiffness of the foams as a consequence of compression. This model leads to a modified scaling law for the flow stress of metallic foams. (C) 2001 Acta Materialia Inc. Published by Elsevier Science Ltd. All rights reserved.
\end{abstract}

Keywords: Aluminum; Foams; Mechanical properties (elastic, plastic); Infiltration

\section{INTRODUCTION}

Recent commercialization of highly porous metal, generally called metallic foam, has led to increased research activity and renewed interest in this class of material [1]. Commercially available metallic foams comprise a range of materials featuring various base metal compositions and pore sizes. The majority of these foams have closed cells that are irregular in size and shape with diameters on the order of one to several millimeters (cf. Figure 1 in Ref. [2]). One notable exception is a comparatively regular but anisotropic open-cell foam marketed under the tradename Duocel (ERG, Oakland CA) [1, 2]. Large, irregular cells typical of commercial foams make testing and interpretation of the mechanical behavior difficult because large specimens are needed to ensure reproducibility. Also, irregular microstructures lead to inhomogeneous deformation and strange (e.g. jagged) flow curves. Thus, metallic foams featuring fine, homogeneous microstructures are useful to capture the intrinsic average deformation behavior of this class of materials.

In this study, we utilize a replication technique to produce open-cell aluminum sponge or foam with relative densities of about $25 \%\left(\approx 0.67 \mathrm{~g} \mathrm{~cm}^{-3}\right)$.

$\dagger$ To whom all correspondence should be addressed. Fax: +41-21-693-4664.

E-mail address: andreas.mortensen@epfl.ch (A. Mortensen)

$\ddagger$ Current address: Department of Materials Science and Engineering, Northwestern University, 2225 N. Campus Dr., Evanston, IL 60208-3108, USA.
These foams feature cells of relatively uniform size and a cell diameter of about $500 \mu \mathrm{m}$. The replication technique consists of three basic steps: preparation of an open-pore pattern, infiltration of the pattern with the desired metal, and subsequent removal of the pattern to produce a metal sponge. The basis of this technique was developed in the 1960s at the PittmanDunn laboratories in Philadelphia, utilizing conventional casting techniques and rock salt as the removable pattern [3]. Compared with foaming techniques which require an alloy or slurry that can resist collapse of the foam structure until it solidifies, this method has the advantage that virtually any alloy can be used to produce metal sponges. Similar techniques have been developed in recent years for both metal and non-metal systems [3-16]. In the present investigation we increase the range of microstructures that can be achieved with replication compared to previous work by presintering salt patterns and also by employing gas-pressure infiltration. This allows the generation of foams with low density and small pore diameters.

The work reported here focuses on the compressive behavior of open-cell, high-purity aluminum sponges having relative densities between 0.2 and 0.27 . We show that the material exhibits significant strain hardening and displays a decrease in stiffness during the initial stages of deformation. A simple analysis of the plastic deformation of these foams is provided to explain these observations and to show how the traditional scaling laws can be modified to account for the intrinsic strain hardening of the bulk metal from which the foam is made. 


\section{EXPERIMENTAL PROCEDURES}

\subsection{Processing}

Pure aluminum foams are produced by infiltration of porous salt patterns and subsequent dissolution of the salt as outlined in Fig. 1; further details are available in $[3,8]$. The pattern is prepared by sintering a packed bed of coarse chemical grade sodium chloride powder. The particles of this high-purity salt are about $500 \mu \mathrm{m}$ in diameter, and their shape is equiaxed, varying somewhat from cubic to spherical depending on the as-received lot.

To produce the pattern, loose salt powder is poured into alumina crucibles that have been coated with boron nitride. The crucible is then tapped until a stable density is achieved. This crucible is placed in a cold furnace and heated to a sintering temperature of $785^{\circ} \mathrm{C}$ in air, followed by furnace cooling. No significant deviations in the properties of the foams have been noted for sintering times ranging from a few hours to $144 \mathrm{~h}$. The temperature of the furnace is controlled to within $\pm 5^{\circ} \mathrm{C}$ over a length of $120 \mathrm{~mm}$, such that homogenous preforms with a length of about 100 $\mathrm{mm}$ and a diameter up to $38 \mathrm{~mm}$ can be produced.

\section{STEP 1: Sintering}

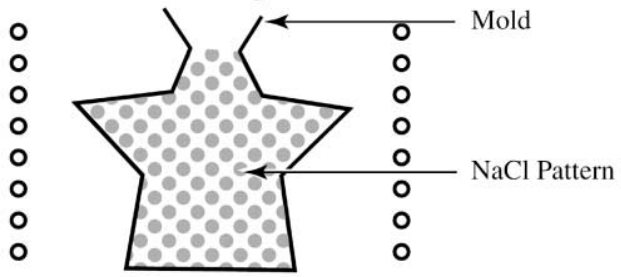

\section{STEP 2: Gas-Pressure Infiltration}

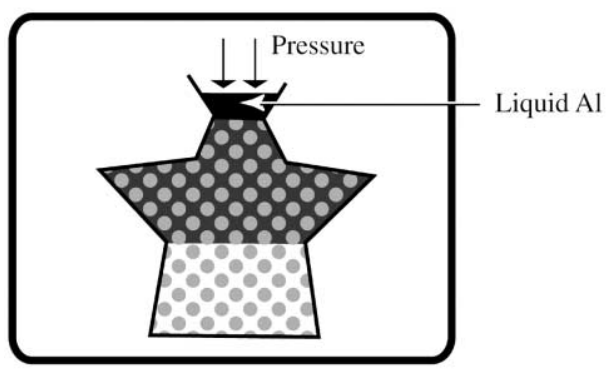

STEP 3: Dissolution

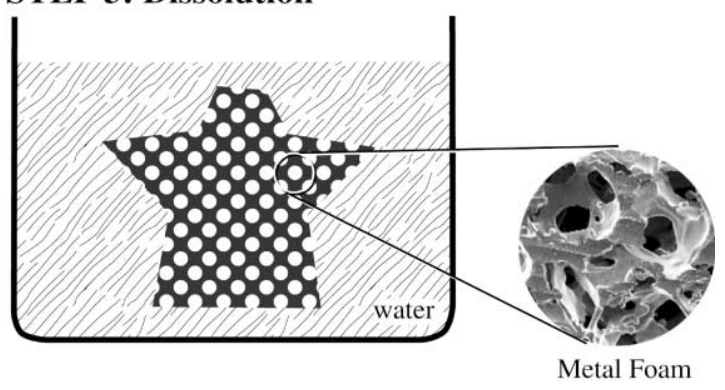

Fig. 1. Schematic of the replication process for the production of metallic foam.
After sintering, the salt pattern is removed, inserted into a similar alumina crucible coated with graphite, and a billet of high-purity $(99.99 \%)$ aluminum is placed on top of the pattern. This assembly is placed in a hot-wall gas-pressure infiltration apparatus, where it is heated to $750^{\circ} \mathrm{C}$ under vacuum. Once the temperature has stabilized, the system is pressurized with argon to a pressure of $0.5 \mathrm{MPa}$, such that the liquid aluminum infiltrates the preform. After infiltration is complete, directional solidification is initiated from the bottom of the crucible upwards by lowering the crucible onto a cold copper chill within the infiltration apparatus. After solidification, the material is removed from the infiltration apparatus and machined to the desired dimensions prior to removal of the salt. The salt is subsequently leached by submersion in distilled water.

\subsection{Microstructural characterization}

The foam density was computed by measuring the mass and dimensions of machined cylindrical compression samples prior to testing. Assuming the bulk aluminum has a density $\rho_{s}$ of $2.698 \mathrm{~g} \mathrm{~cm}^{-3}$, the relative density of the foam (or volume fraction metal $\equiv \rho / \rho_{s}$ where $\rho$ is the density of the foam) can be determined to a precision of $0.1 \mathrm{vol} \%$ and varies within a casting by less than $1 \mathrm{vol} \%$. Macroscopically flat surfaces of foam were prepared by machining or polishing prior to removal of the pattern. After subsequent dissolution of the salt, the microstructure (or more accurately the architecture) of the foam is revealed by examining these surfaces with the aid of a scanning electron microscope.

\subsection{Mechanical testing}

A screw-driven universal testing machine was used for compressive testing. The spherically aligned platens were coated with Teflon to reduce friction with the cylindrical compression specimens, which are nominally $20 \mathrm{~mm}$ in length and $20 \mathrm{~mm}$ in diameter. Cylindrical specimens $20 \mathrm{~mm}$ in length and $10 \mathrm{~mm}$ in diameter were also tested and resulted in the same flow curve. Thus, the larger diameter was chosen as a compromise between optimizing the resolution of the load and the number of samples per casting. A constant cross-head speed of $0.005 \mathrm{~mm} \mathrm{~s}^{-1}$ was used for all tests, corresponding to an initial strain rate of $2.5 \times 10^{-4} \mathrm{~s}^{-1}$. The deformation of the foam was determined to a resolution of $1 \mu \mathrm{m}$ from the crosshead displacement of the machine corrected for the compliance of the load frame and load cell. This compliance was measured before and after each test.

The initial stiffness of the foam was determined from the linear region (maximum slope) portion of the nominal stress-strain response upon reloading after unloading near a nominal strain of $0.2 \%$. In addition, regular unload-reload cycles were used to monitor the evolution of the stiffness of the foam as a function of strain: all stiffness values reported here are determined from the reloading portion of the 
unload-reload cycle. The nominal stress at $2 \%$ strain is used to quantify the yielding behavior of these pure aluminum foams.

The electrical resistance across the foam was also monitored during compression of several specimens. A constant current $(1.5 \mathrm{~A})$ was passed through the specimen via copper platens and the potential drop was measured between two pins that pierced through the diameter of the specimen. In this way the change in potential could be measured to within $0.1 \%$ while under load. The resistivity of a $26 \%$ dense undeformed foam is about $19 \mu \Omega \mathrm{cm}$.

\section{RESULTS}

SEM micrographs of the as-cast foam reveal a uniform architecture that clearly demonstrates the detailed replication of the salt pattern structure (Fig. 2). Facets on the salt can be replicated as well as pits that apparently existed on the salt grains prior to infiltration, possibly as a consequence of thermal etching during sintering [17] (these infiltrated pits become metal protrusions after dissolution of the salt). The foam consists of nonperiodic arrays of struts (or beams) with an hour-glass shape that meet at large irregular nodes. The relative density of the foams varies from about 0.20 to 0.27 and is difficult to control within this range (although the variation within each casting is small, being less than $1 \mathrm{vol} \%$ ). The average density is partially determined by the shape of the salt grains, which, as mentioned, varied somewhat from lot to lot.

The stress-strain response of several foams with different densities is presented in Fig. 3. The initial loading of the foam displays a softer response than upon unloading. A clearly identifiable plateau stress is not observed in these foams; rather, the foam continuously hardens at all strains, and exhibits an inflection point in the flow stress at approximately $10 \%$ strain. At strains lower than $10 \%$, the flow stress can be approximated by a simple power law in nominal stress $s$ and nominal strain $e$ of the form $s \propto e^{n}$ where $n \approx 0.26$ [Fig. 3(b)].

The initial stiffness $E_{0}$ and flow stress at $2 \%$ strain of all tested samples (representing 10 castings) are plotted as a function of the relative density in Figs. 4 and 5, respectively. The evolution of stiffness with strain for several foam densities is given in Fig. 6: as the compressive strain increases, the stiffness initially decreases slightly, then increases rapidly after some critical strain in the vicinity of $15 \%$, i.e., near the inflection of the stress-strain curves. As the sample deforms, the relative electrical potential drop $\left(V / V_{0}\right)$ shows a continuous decrease as a function of compressive strain (Fig. 7).

\section{MODEL}

We consider the microscopic deformation of a tetrahedral arrangement of beams, a basic unit for (a)

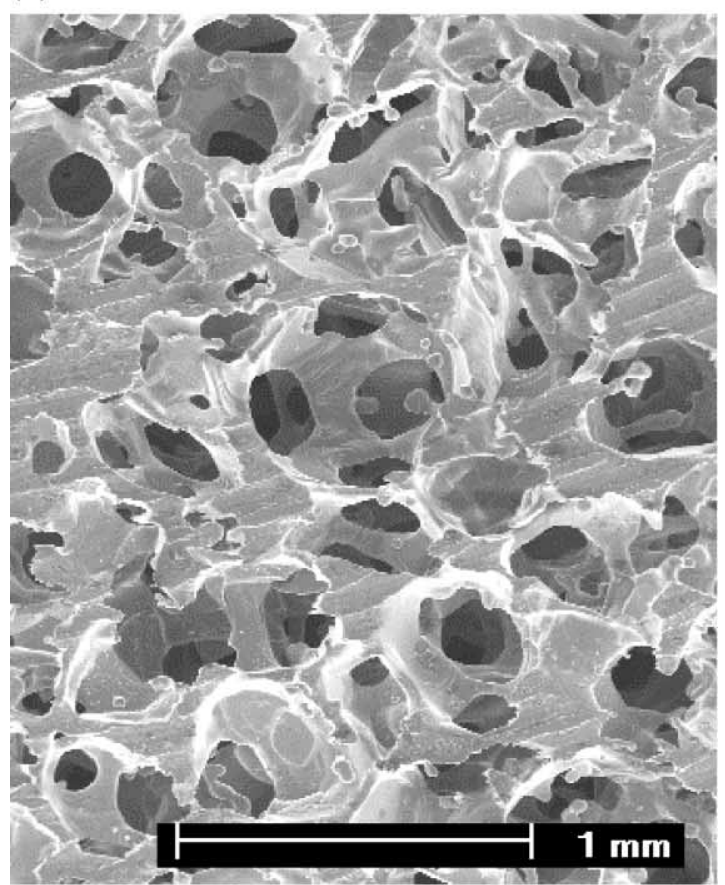

(b)

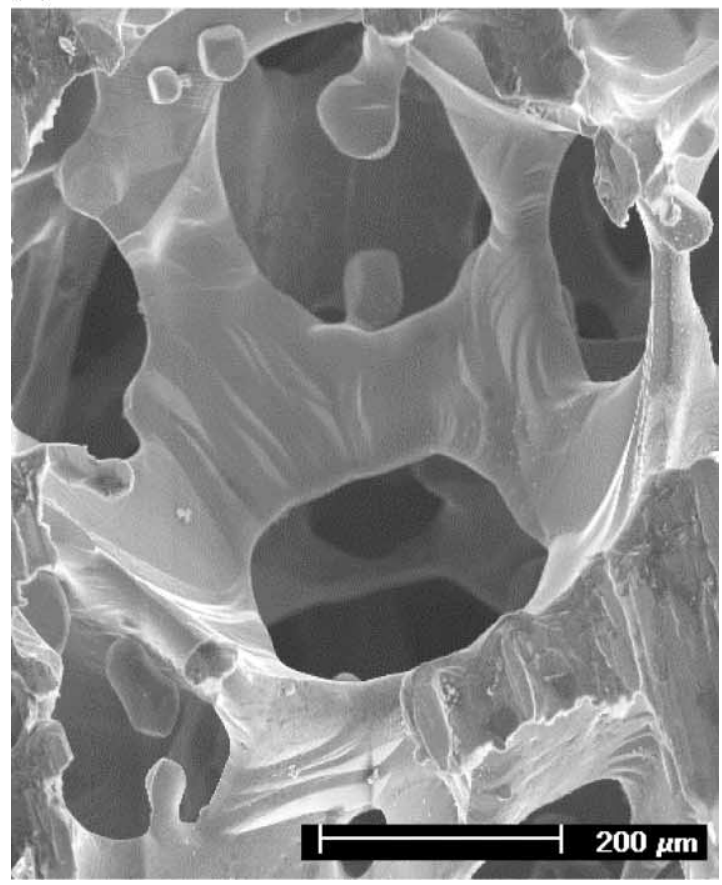

Fig. 2. (a) SEM micrograph of pure aluminum foam $\left(f_{0}=0.21\right)$ produced by replication. (b) SEM micrograph of pure aluminum foam $\left(f_{0}=0.21\right)$ produced by replication, showing the shape of a node and associated struts, and the replication of thermal etch pits.

open-celled porous networks which has been studied before in the literature [18-20]. In the present context, one can rationalize this unit considering the packing of equal-sized spheres, as these foams are produced by infiltration of partially sintered equiaxed grains. 
(a)

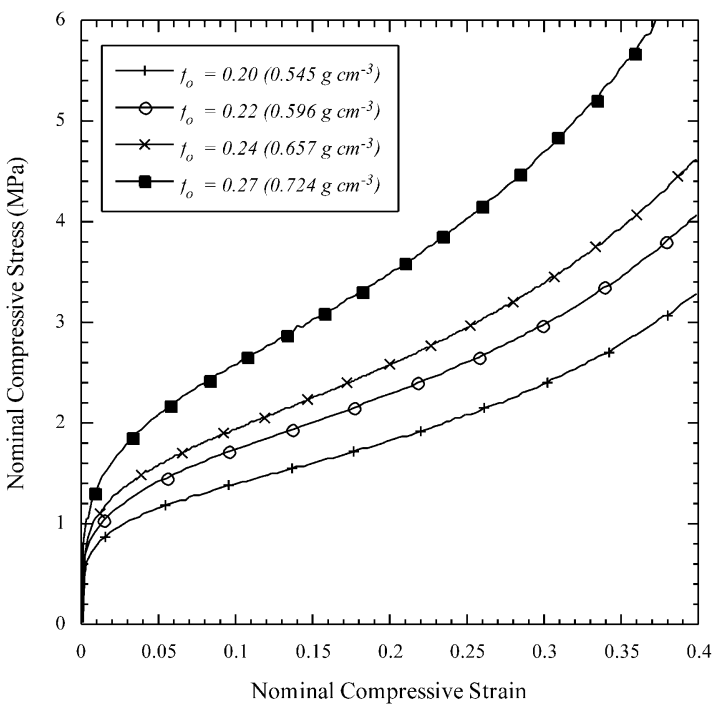

(b)

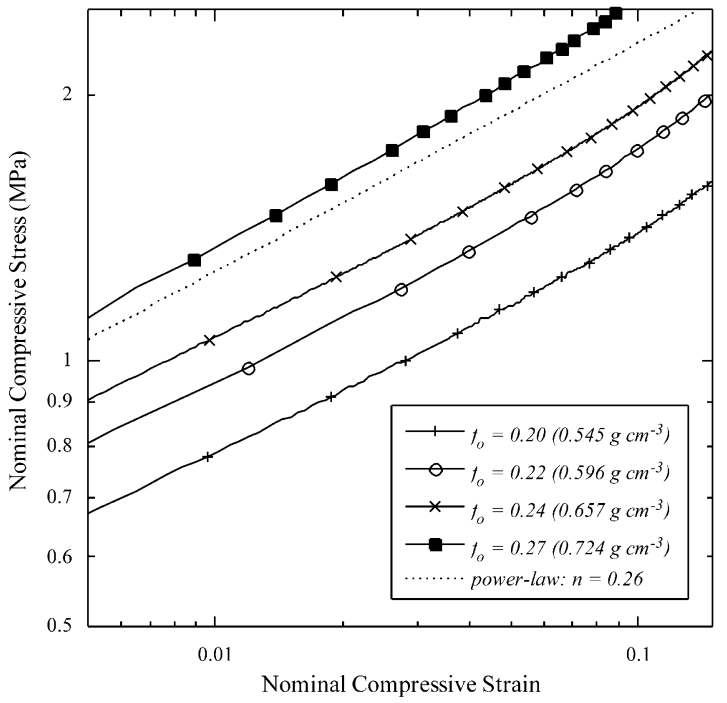

Fig. 3. (a) The compressive response of pure aluminum foam of various densities demonstrating the strain hardening character of these foams. (b) The compressive response of pure aluminum foam of various densities at low strains. The (straight) dotted line represents a power law relationship between the nominal stress and nominal strain with an exponent of 0.26

The interstices between four close-packed spheres create four struts or beams connected in the center of this cluster. Each beam is bounded by three spheres and thus has an idealized triangular cross-section. For equal-sized spheres in this configuration, the angles between each of the four beams must be equal to the tetrahedral angle:

$$
\alpha \equiv \cos ^{-1}(-1 / 3)=109.47^{\circ}
$$

For simplicity we analyze the response of tetrahedral elements stacked periodically to form a hexagonal structure and loaded axially, as illustrated in Fig.

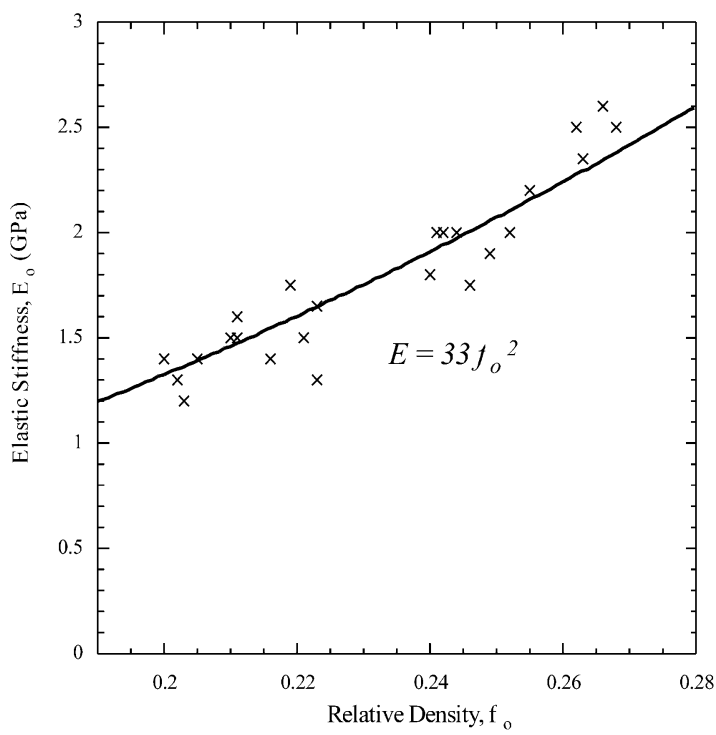

Fig. 4. The elastic stiffness of replicated aluminum foam as a function of density.

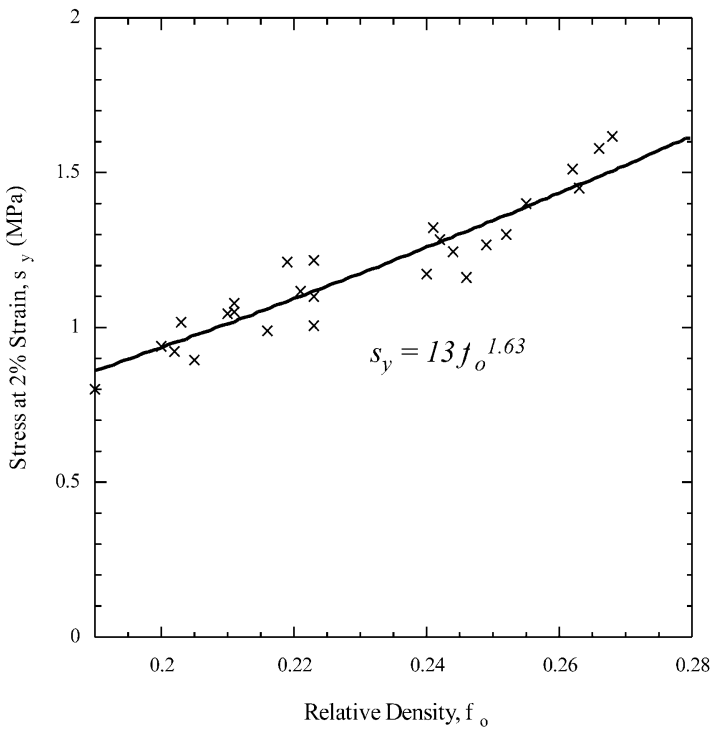

Fig. 5. The stress at $2 \%$ nominal strain for replicated pure aluminum foam and the fit to equation (14).

8. This is clearly an idealization; however, it is amenable to a simple analytical treatment that captures the basic compressive response of the present opencelled foams.

\subsection{Basic relations}

Consider the basic (undeformed) unit as shown in Fig. 8(a): four beams meet at a node, each beam has a length $L$, one beam is aligned along the axis of a hexagon, and the three inclined beams are symmetric making an angle $\phi_{0}$ with the axial beam. A volumetric ratio of the four beams (assuming negligible volume in the node) to the hexagonal space for this unit cell leads to an expression for the initial relative density 


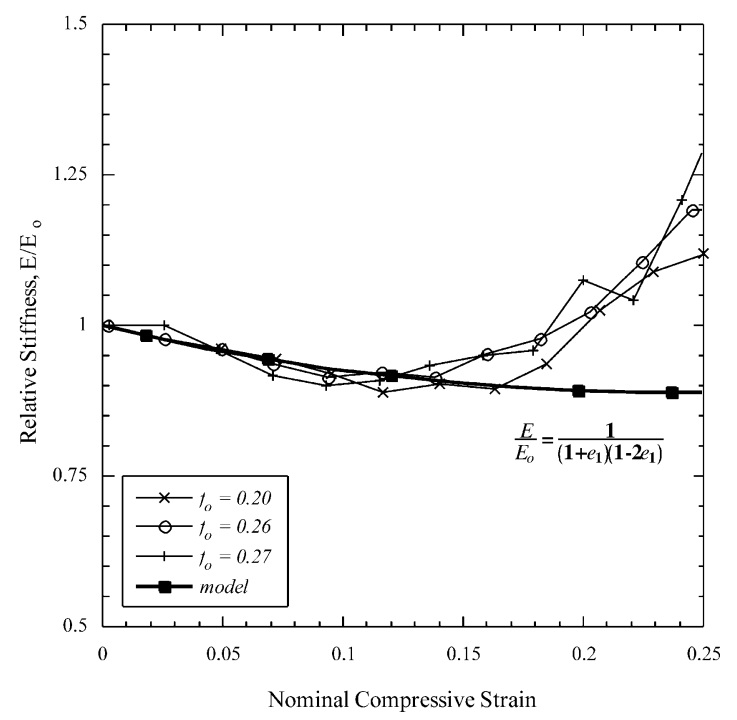

Fig. 6. The evolution of stiffness for several open-cell aluminum foams and the predicted evolution after equation (22).

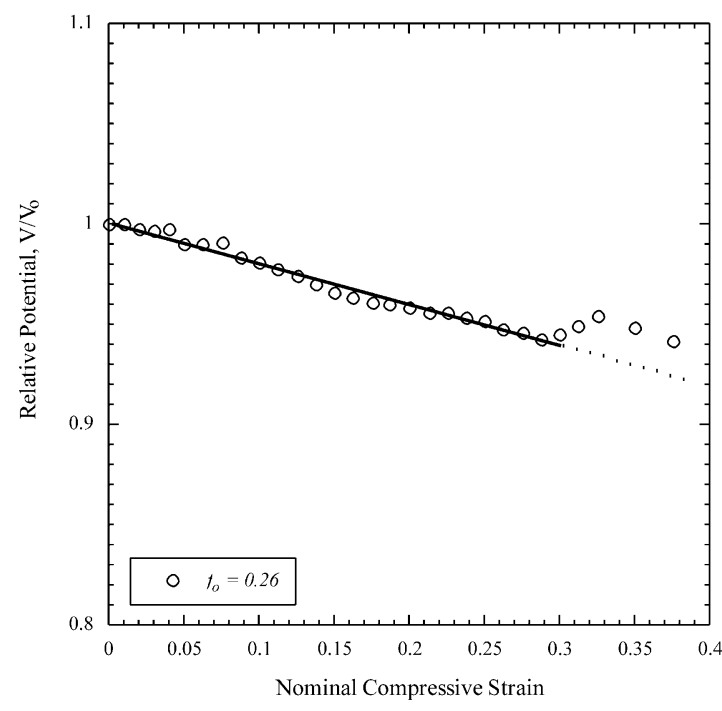

Fig. 7. The electrical resistivity of the foam decreases continuously as the material is compressed.

(or volume fraction) $f_{0}$ of the porous material which reads

$$
f_{0}=C_{f 0}\left(\frac{B}{L}\right)^{2}
$$

where $C_{f 0}$ is a geometrical constant based on the beam geometry and $B$ is a characteristic dimension related to the cross-section of the beams.

As a consequence of the assumed uniaxial loading along the axial beam, each of the three inclined beams carries one-third of the applied load. At small strains, the nominal stress $s_{1}$ and strain $e_{1}$ of the foam in the loading direction can be written in terms of the dimensions of the volumetric element $\left(a_{0}\right.$ and $h_{0}$ are defined in Fig. 8), the applied load $P_{1}$, and the deflection $\delta_{1}$ along the loading axis:

$$
\begin{gathered}
s_{1}=\frac{P_{1}}{a_{0}}=\frac{P_{1}}{\frac{3 \sqrt{3}}{2}\left(L \sin \phi_{0}\right)^{2}} \\
e_{1}=\frac{\delta_{1}}{h_{0}}=\frac{\delta_{1}}{L\left(1-\cos \phi_{0}\right)}
\end{gathered}
$$

Since bending is generally the dominant deformation mechanism in open-cell foams [2], the axial deflection is neglected in all of the beams. From symmetry we can then consider a single inclined beam as shown in Fig. 9. The load normal to the beam $F$ and the beam deflection $\Delta$ along this direction are related to $P_{1}, \delta_{1}$ and the average angle $\phi$ made by the beam with the loading axis:

$$
\begin{gathered}
F=\frac{P_{1}}{3} \sin \phi \\
\delta_{1}=\Delta \sin \phi
\end{gathered}
$$

We first describe the plastic deformation of this characteristic beam using elasto-plastic beam theory and relate its deformation to the simple volumetric unit under uniaxial loading. The flow stress of the foam is shown to be related to the flow stress of the bulk solid, assuming the latter exhibits plastic flow described by power-law hardening. A simple approximation is then used to complement this description with predictions for the elastic stiffness and its evolution as a consequence of deformation.

\subsection{Initial plastic deformation}

Due to the nodes, the ends of our characteristic beam are fixed such that loading produces a moment $M$ that varies along the length $L$ of the beam as

$$
M=\frac{F}{2}(L-2 y)
$$

where $y$ is the position with respect to the fixed end loaded in the negative $x$-direction as shown in Fig. 9. This moment is carried by the stress $\sigma(x)$ in the crosssectional area $A$ of the beam:

$$
M=\int_{A} x \sigma \mathrm{d} A
$$

The corresponding strain $\varepsilon$ within the beam varies through the cross-section as the ratio of the distance 

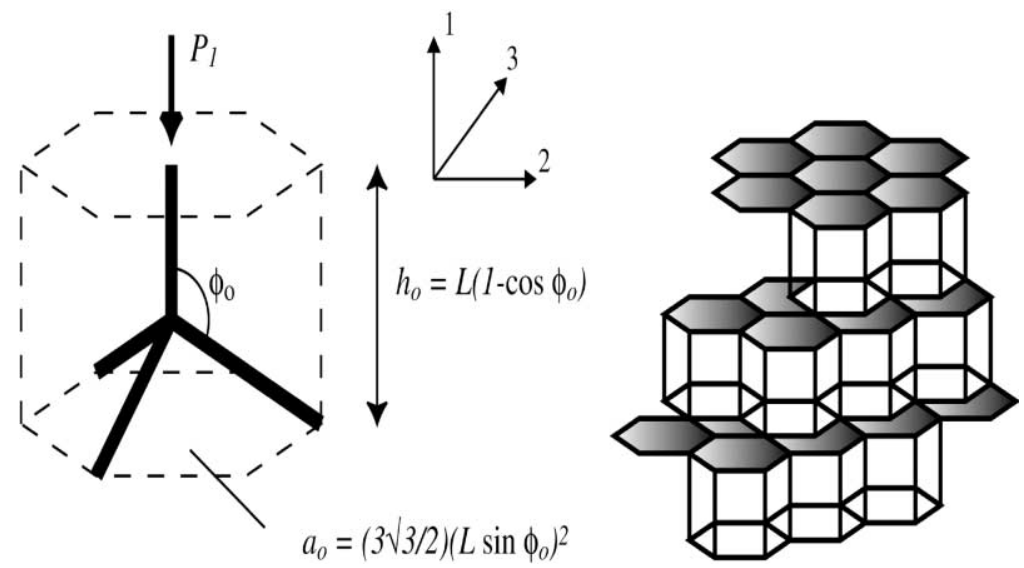

Fig. 8. Schematic of (a) the tetrahedral unit used for modeling deformation in replicated foam and (b) the periodic stacking of the tetrahedrons.
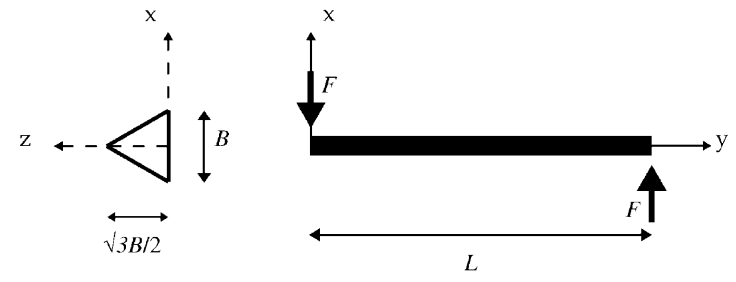

Fig. 9. Schematic of the characteristic beam with fixed ends and the assumed loading configuration.

from the neutral axis $x$ to the radius of curvature of the beam $R$ :

$$
\varepsilon=x / R
$$

(tension is positive, and $R$ is positive where the beam is curved downwards). We consider a bulk material with a power-law stress-strain relationship of the form

$$
\sigma=\sigma_{0} \varepsilon^{n}
$$

where $\sigma_{0}$ and $n$ are constants. Combining equations (7)-(10) and integrating, one obtains for small deflections

$$
\frac{1}{R}=\frac{\mathrm{d}^{2} \Delta}{\mathrm{d} y^{2}}=C^{\prime}\left[\frac{F}{\sigma_{0}} \frac{(L-2 y)}{B^{3}}\right]^{1 / n} \frac{1}{B}
$$

where $C^{\prime}$ is a geometrical constant that also depends on $n$. The fixed ends of the beam require that

$$
\Delta(y=0)=0
$$

$$
\frac{\mathrm{d} \Delta}{\mathrm{d} y}(y=0)=0
$$

The beam is symmetric about its midpoint, thus the maximum displacement $\Delta_{\max }$ of the beam with respect to a fixed end is twice the displacement at the middle $y=L / 2$. By integration of equation (11), $\Delta_{\max }$ reads

$$
\Delta_{\max }=\frac{C^{\prime}}{2\left(\frac{1}{n}+2\right)}\left[\frac{F L}{\sigma_{0} B^{3}}\right]^{1 / n} \frac{L^{2}}{B}
$$

For small strains $\phi \approx \phi_{0}$, the uniaxial nominal stressnominal strain response of the foam can then be expressed by combining equations (3)-(6) with equation (13):

$$
s_{1}=C \sigma_{0} f_{0}^{(3+n) / 2} e_{1}^{n}
$$

$C$ is a geometrical constant related to $C^{\prime}$; both constants are given in Appendix A for triangular and square cross-sections. The specifics of the foam architecture (beam arrangement and cross-section) are contained in the constant $C$; thus this relationship is general for open-celled foams that deform by beam bending and that are made of material described by power-law hardening. This equation, however, is restricted to small deformations so that $C$ indeed remains a constant.

\subsection{Elastic response}

To determine the elastic response of this model foam at any point in the strain history, we idealize the plastically bent beams as remaining straight, as if 
all deformation were concentrated at the node only (as is the case for an ideally plastic material, since this is the point of maximum moment). In contrast to Section 4.2, potentially large deformations can then be characterized by a changing angle $\phi$ between the three inclined struts and the axial strut (depicted in Fig. 8 for $\phi=\phi_{0}$ ). The nominal plastic strain in the axial direction $e_{1}$ is then related uniquely to $\phi$ and the initial angle $\phi_{0}$ by

$$
e_{1}=\frac{1-\cos \phi}{1-\cos \phi_{0}}-1
$$

At any point in the loading history of the foam, all of elastic deformation is assumed to result from simple bending in the three symmetric inclined beams. The elastic deflection of the foam can then be derived from simple elastic beam theory: the elastic deflection $\Delta_{\mathrm{el}}$ of a beam has the general form

$$
\Delta_{\mathrm{el}}=K \frac{F L^{3}}{E_{\mathrm{s}} I}
$$

where $E_{\mathrm{s}}$ is the Young's modulus of the beam (bulk) material, $I$ is the moment of inertia and $K$ is a constant that depends on boundary and loading conditions. By substituting equations (3)-(6) into equation (16), the relationship between the nominal stress and nominal elastic strain for the hexagonal unit in Fig. 8 is

$$
s_{1}=\frac{2 \sqrt{3}}{3 K} \frac{\left(1-\cos \phi_{0}\right)}{\left(\sin \phi_{0}\right)^{2}(\sin \phi)^{2}} \frac{I}{L^{4}} E_{\mathrm{s}} e_{1, \mathrm{el}}
$$

at a fixed plastic strain where $e_{1, \mathrm{el}}$ is the elastic portion of the strain (from $\Delta_{\mathrm{el}}$ ). The geometrical ratio $I / L^{4}$ can be expressed in terms of the relative density by noting that the moment of inertia depends on the geometry of the cross-section of the beam and that, for a symmetric beam, $I$ can be written in terms of the characteristic dimension (or thickness) of the beam $B$ as

$$
I=C_{l} B^{4}
$$

where $C_{I}$ is a constant. By substituting equations (2) and (18) into equation (17), the (nominal) elastic stiffness $E$ at a specific plastic strain can be expressed as a function of the relative density:

$$
E=\left(\frac{\partial s_{1}}{\partial e_{1, \mathrm{el}}}\right)_{e_{1}}=C_{E}\left(\frac{\sin \phi_{0}}{\sin \phi}\right)^{2} E_{\mathrm{s}} f_{0}^{2}
$$

where the constants are collected into $C_{E}$ as given in Appendix A. The initial foam stiffness $\left(E_{0}\right.$ at $\left.\phi=\phi_{0}\right)$ is then

$$
\frac{E_{0}}{E_{\mathrm{s}}}=C_{E} f_{0}^{2}
$$

Additionally, with $\phi_{0}=\alpha$ it can be shown that

$$
\left(\frac{\sin \phi}{\sin \phi_{0}}\right)^{2}=\left(1+e_{1}\right)\left(1-2 e_{1}\right)
$$

Combining the prior three equations provides a relationship for the evolution of the stiffness at any point in its deformation history (i.e. as a function of strain):

$$
\frac{E}{E_{0}}=\frac{1}{\left(1+e_{1}\right)\left(1-2 e_{1}\right)}
$$

\section{DISCUSSION}

\subsection{Elastic properties}

Many analytical models have been proposed for the elastic stiffness of cellular materials, and with each there is associated a specific set of assumptions about the architecture of the foam and appropriate deformation mechanisms [18, 19, 21-26]. In summary, for open-cell foams where elastic deformation is dominated by stretching of the beams, the stiffness is a linear function of the relative density. Deformation of open-cell metal foams, however, is dominated by bending and models predict that the elastic stiffness will exhibit a square dependence on the relative density as was found in Section 4.3.

Gibson and Ashby use a phenomenological approach to obtain a relation identical to equation (20) and estimate $C_{E}=1$ from experimental results for several types of cellular materials [23]. Other analyses also arrive at $C_{E} \sim 1[18,22,24]$. Warren and Kraynik develop equation (20) using the same tetrahedral element but randomly oriented and assuming affine deformation. They find that $C_{E}$ is a function of the volume fraction solid, which varies, for triangular beams, from 1.1 as the volume fraction goes to zero, to $2 / 3$ at a relative density of 0.25 [19]. The present derivation yields a constant value $C_{E}=4 / 3$ for triangular beams (Warren and Kraynick's calculations show that the tetrahedron stiffness is at a maximum for the orientation that we assume).

The experimentally measured elastic stiffness of the aluminum sponge produced in this study, indeed, follows the basic functionality of equation (20), (Fig. 4). These data, however, suggest a value of $C_{E}$ closer to 0.5 . Since macroscopic defects are not present in the foams studied here, this discrepancy is attributed to the non-ideal microscopic architecture of the foam, as evident from micrographs (Fig. 2). The present foam is made up of interconnected beams, but the cross-sections of these beams are not uniform and their length to thickness ratio is on the order of 2; as 
a consequence the nodes contain a significant portion of the material. Additionally, surface roughness and protrusions in the foam (Fig. 2) represent a volume of material that is "lost" in a structural sense since it carries little or no load. Values of $C_{E}$ lower than predicted by theory are not unusual, and several other studies, both theoretical and experimental, have demonstrated that stiffness strongly depends on the architecture of foams [27-35].

\subsection{Low-strain plastic deformation}

The measured evolution of stiffness with plastic deformation agrees well with the analysis in Section 4.3: equation (22) quantitatively captures the decrease in the normalized stiffness of the foam up to strain of about $15 \%$ (Fig. 6). The observed evolution of the normalized stiffness at strains below $15 \%$ is also independent of the density of the foam, as predicted. Thus, the change in stiffness can be attributed to the assumed geometrical evolution of the structure of the foam.

The stress strain response is related to the deformation and hardening of the structural elements themselves. Figure 3(b) shows that a power law describes well the stress-strain response for $0.5 \%<e_{1}<10 \%$ and that $n \approx 0.26$ for all foams studied here. The flow stress of bulk aluminum is dependent on parameters such as level of impurities, grain size, cell size and dislocation density [36-38], but $n \approx 0.26$ is consistent with reports in the literature for high-purity bulk aluminum at strains greater than about 3\% [39-42]. Although metallic foams studied in the literature often display a flow curve characterized by a constant flow stress over a wide range of strains (the so-called plateau stress) $[1,2,23,31,43,44]$, strain hardening similar to that observed in this study is also evident in a few prior studies of foams made from relatively ductile metals such as nominally pure aluminum and low-alloyed aluminum [43-47]. The experimental data, thus, agree at low strains with the analysis in Section 4.2 and are consistent with earlier results.

Ashby and Gibson use dimensional analysis to show that for open cell foams the flow (plateau) stress $s_{\mathrm{pl}}$ can be expressed in terms of the foam density and the yield strength $\sigma_{\mathrm{y}}$ of the bulk material as

$$
s_{\mathrm{pl}}=C_{\mathrm{y}} \sigma_{\mathrm{y}} f_{0}^{3 / 2}
$$

where $C_{\mathrm{y}}$ is a constant about 0.3 [23]. This relation assumes deformation by the formation of plastic hinges at the nodes, as expected for a perfectly plastic material. In the limit as $n \rightarrow 0$, the analysis in Section 4.2 predicts (as it should) that the curvature of a deflecting beam is localized near the nodes (fixed ends), and that equation (14) reduces to equation (23) with $C \sim 0.47$. This value agrees with the experimentally determined value proposed by Ashby and Gibson for open-cellular (non-metal) materials.

For a strain-hardening metal of finite $n$, the present analysis predicts a relationship [equation (14)] between flow stress and foam density that is qualitatively similar to equation (23); however, an additional factor $f_{0}^{n / 2}$ results from strain hardening of the bulk material (yielding also the proper limiting behavior as $n \rightarrow 1$ ). Fitting the measured yield stress (defined as the flow stress at $2 \%$ strain) to the relative density raised to the $1.63(=(3+n) / 2)$ power results in

$$
s_{\mathrm{y}}[\mathrm{MPa}] \approx 13 f_{0}^{1.63}
$$

which describes the data well (Fig. 5). The flow stress of these foams can thus be expressed in terms of density and strain as

$$
s_{1}[\mathrm{MPa}] \approx 36 f_{0}^{1.63} e_{1}^{0.26}
$$

Microhardness measurements on ligaments of the pure aluminum foams produced in this study indicate a yield stress of about $75 \mathrm{MPa}$. One-third of this yield stress $\left(C_{\mathrm{y}} \sigma_{\mathrm{y}}\right)$ is approximately twice the factor experimentally determined in equation (24) when corrected by the factor $f_{0}^{n / 2}$. In other words, approximately the same "knock-down" factor is observed for the measured stiffness and the measured yield stress when compared to the relations determined here or by Gibson and Ashby. While there is no obvious physical reason that the stiffness and yield stress should be corrected by the same factor, this reinforces the conclusion that these differences arise from the same source: the real internal architecture of the foam (with its thick nodes and protrusions) versus the idealized structure of straight beams assumed in the models.

It should also be noted that the observed yield stress of $75 \mathrm{MPa}$ is significantly higher than that of annealed 99.99\% pure aluminum (around $10 \mathrm{MPa}$ $[38,42,48])$. In the absence of significant solubility of $\mathrm{NaCl}$ in aluminum, the hardening of the aluminum is most likely dislocational and due to differential thermal shrinkage between the salt and the aluminum during cooldown from processing temperatures [49, 50].

\subsection{High-strain plastic deformation}

At strains above $10 \%$, the measured flow stress clearly departs from the power-law behavior predicted for small strain plasticity in Section 4.2. Although these deviations [Fig. 3(b)] develop slowly over a range of strains, they become marked at approximately the same strain as the measured minimum in stiffness. After this minimum, the stiffness begins increasing rapidly (Fig. 6). In comparison, equation (22) predicts a minimum in the stiffness at $25 \%$ compressive strain, with a subsequent increase in stiffness at a rate similar to the decrease. We attribute the deviations between the model and experiment for both the flow curve and stiffness to a common source: densification (i.e. the formation of contacting elements in the structure that carry additional load in compression). 
The strain at which the stiffness begins to increase and the inflection in the flow curve are greater for foams with a lower density, Figs 3(b) and 6. This indicates that structural interactions become important earlier in the strain history for denser foams, as is generally observed for densification [51, 52]. The presence of densification is further supported by the observed decrease in the electrical potential with deformation (Fig. 7). This implies that beam-tobeam contact points are created early in the deformation history and continue to be formed as deformation proceeds, since neither plastic nor elastic deformation (i.e. bending of struts or the formation of a plastic hinge in a beam) itself should cause a change in the potential drop across the foam. This gradual densification also contributes, in part, to the observed strain hardening, however, this contribution is deemed to be small at strains below 10 to $15 \%$, since the stiffness, which is also sensitive to densification, initially decreases, cf. Fig. 3 and Fig. 6.

Similar tendencies with regard to the evolution of stiffness with straining have been noted in the literature for commercially available aluminum foams: for both closed-cell foams [44, 46] and open-cell foams [53] the stiffness decreases upon straining, reaches a minimum then begins to increase. An analogy may then exist in closed-cell foams for the evolution of stiffness with strain as described in Section 4.3 and based on a changing geometrical relationship between structural elements. Such an interpretation in closedcell foams, however, may be difficult to formulate in the presence of more complex deformation mechanisms such as localized structural collapse [54].

\section{CONCLUSIONS}

Open-cell aluminum foams or sponges produced by replication with relative densities on the order of 0.25 feature a macroscopically homogeneous structure. The microscopic architecture consists of irregular struts connected at large nodes and the open porosity in the salt pattern is clearly replicated including facets on the salt surface and small pits in the salt grains.

These foams deform homogeneously in compression, exhibiting the following general characteristics:

- a low-strain response that is not elastic from the onset, but which, upon unloading after a small strain increment, displays a linear elastic response with a stiffness proportional to the square of the relative density;

- power-law strain hardening at low strains with an exponent close to $1 / 4$, a value typical of strained pure aluminum;

- a yield stress that depends on the relative density raised to a power greater than 1.5 ;

- an increase in stiffness and an inflection in the flow stress at approximately the same strain; this strain is on the order of $10 \%$ and increases for foams with lower density.

A simple unit-cell analysis is proposed which accounts for the observed density dependence of the stiffness $\left(f_{0}^{2}\right)$ and flow stress $\left(f_{0}^{(3+n) / 2}\right)$ of the foam. This analysis also accurately describes at low strains the strain dependence of the flow stress and the observed change in stiffness with straining. The deviations from predictions at strains higher than about $10 \%$ are interpreted as being a consequence of densification.

Acknowledgements-This work was funded by core funding in the Laboratory of Mechanical Metallurgy at EPFL and the Swiss National Science Foundation project no. 21-58839.99. The authors would like to thank Dr. L. Weber for assistance with these measurements, and J.-F. Despois for assistance with the manuscript and useful suggestions.

\section{REFERENCES}

1. Ashby, M. F., Evans, A., Fleck, N. A., Gibson, L. J., Hutchinson, J. W. and Wadley, H. N. G., Metal Foams: A Design Guide. Butterworth Heinemann, Boston, MA, 2000.

2. Gibson, L. J., Аnnu. Rev. Mater. Sci., 2000, 30, 191.

3. Polonsky, L., Lipson, S. and Markus, H., Modern Cast., 1961, 39, 57-71.

4. Kuchek, H. A. Method of making porous metallic article. US patent 3236 706, 1966.

5. Pekala, R. W. and Hopper, R. W., J. Mater. Sci., 1987, 22, 1840.

6. Fitzgerald, T. J., Michaud, V. J. and Mortensen, A., J. Mater. Sci., 1995, 30, 1037.

7. Ma, L., Song, Z. and He, D., Scripta mater., 1999, 41, 785.

8. San Marchi, C., Despois, J. F. and Mortensen, A., in Metal Matrix Composites and Metallic Foams, Euromat 99, Proc. Conf., Munich, ed. T. W. Clyne and F. Simancik. DGM/Wiley-VCH, 1999, p. 34.

9. Wagner, I., Hintz, C. and Sahm, P. R., in Metal Matrix Composites and Metallic Foams, Euromat 99, Proc. Conf., Munich, ed. T. W. Clyne and F. Simancik. DGM/WileyVCH, 1999, p. 40.

10. Mohr, U. and Bleck, W., in Metal Matrix Composites and Metallic Foams, Euromat 99, Proc. Conf., Munich, ed. T. W. Clyne and F. Simancik. DGM/Wiley-VCH, 1999, p. 28

11. Yamada, Y., Shimojima, K., Sakaguchi, Y., Mabuchi, M., Nakamura, M.Asahina, T. et al., Mater. Sci. Eng. A, 1999, 272, 455.

12. Yamada, Y., Shimojima, K., Sakaguchi, Y., Mabuchi, M., Nakamura, M.Asahina, T. et al., J. Mater. Sci. Lett., 1999, 18, 1477.

13. Banhart, J., Adv. Eng. Mater., 2000, 2, 188

14. Yamada, Y., Shimojima, K., Sakaguchi, Y., Mabuchi, M., Nakamura, M.Asahina, T. et al., Mater. Sci. Eng. A, 2000, 280, 225.

15. Yamada, Y., Shimojima, K., Sakaguchi, Y., Mabuchi, M., Nakamura, M.Asahina, T. et al., Adv. Eng. Mater., 2000, $2,184$.

16. Lu, T. J., Chen, F. and He, D., J. Acoust. Soc. Am., 2000, 108, 1697.

17. Budke, J., J. Am. Ceram. Soc., 1968, 51, 238.

18. Menges, G. and Knipschild, F., Polym. Eng. Sci., 1975, $15,623$.

19. Warren, W. E. and Kraynik, A. M., J. Appl. Mech., 1988, $\mathbf{5 5}, 341$. 
20. Ford, C. M. and Gibson, L. J., Int. J. Mech. Sci., 1998, 40, 521.

21. Warren, W. E. and Kraynik, A. M., J. Appl. Mech., 1991, 58, 376.

22. Choi, J. B. and Lakes, R. S., Int. J. Mech. Sci., 1995, 37,51 .

23. Gibson, L. J. and Ashby, M. F., Cellular Solids: Structure and Properties, 2nd ed. Cambridge University Press, Cambridge, 1997.

24. Zhu, H. X., Knott, J. F. and Mills, N. J., J. Mech. Phys. Solids, 1997, 45, 319.

25. Grenestedt, J. L., Int. J. Solids Struct., 1999, 36, 1471.

26. Christensen, R. M., Int. J. Solids Struct., 2000, 37, 93.

27. Sugimura, Y., Meyer, J., He, M. Y., Bart-Smith, H., Grenestedt, J. and Evans, A. G., Acta mater., 1997, 45, 5245.

28. Simone, A. E. and Gibson, L. J., Acta mater., 1998, 46, 2139.

29. Simone, A. E. and Gibson, L. J., Acta mater., 1998, 46, 3929.

30. Grenestedt, J. L., J. Mech. Phys. Solids, 1998, 46, 29.

31. Miyoshi, T., Itoh, M., Mukai, T., Kanahashi, H., Kohzu, H.Tanabe, S. et al., Scripta mater., 1999, 41, 1055.

32. Grenestedt, J. L., Scripta mater., 1999, 40, 71.

33. Grenestedt, J. L., Int. J. Mech. Sci., 2000, 42, 1327.

34. Badiche, X., Forest, S., Guibert, T., Bienvenu, Y., Bartout, J. -D.Ienny, P. et al., Mater. Sci. Eng. A, 2000, 289, 276.

35. Nieh, T. G., Higashi, K. and Wadsworth, J., Mater. Sci. Eng. A, 2000, 283, 105.

36. Karnes, C. H. and Ripperger, E. A., J. Mech. Phys. Solids, 1966, 14, 75.

37. Holt, D. L., Babcock, S. G., Green, S. J. and Maiden, C. J., Trans. ASM, 1967, 60, 152.

38. Fujita, H. and Tabata, T., Acta metall., 1973, 21, 355.

39. Lindholm, U. S., J. Mech. Phys. Solids, 1964, 12, 317.

40. Yoshida, S. and Nagata, N., Trans. Natl. Res. Inst. Met., 1967, 9, 20.

41. Kocks, U. F., J. Eng. Mater. Tech., 1976, 98, 76.

42. Kovacs-Csetenyi, E., Horvath, M., Chinh, N. Q. and Kovacs, I., Phys. Stat. Sol. A, 1998, 166, 805.

43. Andrews, E., Sanders, W. and Gibson, L. J., Mater. Sci. Eng. A, 1999, 270, 113.

44. McCullough, K. Y. G., Fleck, N. A. and Ashby, M. F., Acta mater., 1999, 47, 2323.

45. Thornton, P. H. and Magee, C. L., Metall. Trans. A, 1975, 6, 1253.

46. Gradinger, R., Simancik, F. and Degischer, H. P., in Int. Conf. Welding Technology, Materials and Materials Testing, Fracture Mechanics and Quality Management, Proc. Conf., Vienna University of Technology, ed. S. Felber, T. Varga and J. L. Zeman. Chytra Druck and Verlag, Vienna, 1997, p. 701.

47. Han, F., Zhu, Z. and Gao, J., Metall. Mater. Trans. A, 1998, 29, 2497.

48. Metals Handbook, Ninth Edition: Volume 2 Properties and Selection: Nonferrous Alloys and Pure Metals. ASM International, Materials Park, OH, 1979.

49. Arsenault, R. J. and Shi, N., Mater. Sci. Eng., 1986, 81, 175

50. Vogelsang, M., Arsenault, R. J. and Fisher, R. M., Metall. Trans. A, 1986, 17, 379.

51. Ashby, M. F., Metall. Trans. A, 1983, 14, 1755.

52. Maiti, S. K., Gibson, L. J. and Ashby, M. F., Acta metall., 1984, 32, 1963.

53. Harte, A. -M., Fleck, N. A. and Ashby, M. F., Acta mater., 1999, 47, 2511.

54. Bastawros, A. -F., Bart-Smith, H. and Evans, A. G., J. Mech. Phys. Solids, 2000, 48, 301.

\section{APPENDIX A}

The relationships developed in Section 4 are specific to the tetrahedral element in a hexagonal unit.
Only slight modifications are needed for other geometrical units. The expressions are general with respect to the cross-section of the beam elements, such that only the numerical value of the constants introduced in Section 4 depend on the cross-section of beam. Here we evaluate these constants for two specific cases: an equilateral triangular cross-section as shown in Fig. 9, and a square cross-section. While all the relations are given in terms of a general angle $\phi_{0}$, the tetrahedral angle defined in equation (1) is used for all numerical evaluations. We should also emphasize that for cross-sections with more than twofold symmetry (such as those assumed here), the moment of inertia about the centroid is independent of orientation and the product of inertia is zero, such that for simple in-plane loading the bending moment has only a single component.

The constants $C_{E}$ and $C$ can be expressed in a form that is independent of the specific cross-section of the beams:

$$
\begin{gathered}
C_{E}=\frac{2 \sqrt{3} C_{I}}{3 K\left(C_{f 0}\right)^{2}} \frac{\left(1-\cos \phi_{0}\right)}{\left(\sin \phi_{0}\right)^{4}} \\
C=\frac{2}{\sqrt{3}\left(\sin \phi_{0}\right)^{3}}\left[\frac{2\left(1-\cos \phi_{0}\right)\left(\frac{1}{n}+2\right)}{\sin \phi_{0}}\right]^{n} \\
\left(\frac{1}{C^{\prime}}\right)^{n}\left(\frac{1}{C_{f 0}}\right)^{(3+n) / 2}
\end{gathered}
$$

In these expressions, $C_{I}, C_{f 0}$, and $C^{\prime}$ depend on the specifics of the beam cross-section. The constant $K$ as mentioned depends on the loading conditions, and for simple symmetric loading at fixed ends, $K=1 / 12$. In general, $C$ depends on the specific geometry of the beams even in the limit as $n \rightarrow 0$, however, for both triangular and square beams $C \sim 0.47$ in this limit.

\section{A.1. Triangular cross-section}

The constant $C_{I}$ equals $\sqrt{3} / 96$ for an equilateral triangle with a side $B$. For the hexagonal unit sketched in Fig. 8 with equilateral triangular beams, $C_{f 0}$ is related to the initial angle $\phi_{0}$ as

$$
C_{f 0}=\frac{2}{3\left(1-\cos \phi_{0}\right)\left(\sin \phi_{0}\right)^{2}}
$$

In this context, the tetrahedral angle is of interest; then $\phi_{0}=\alpha$ and $C_{f 0}=9 / 16$. Following the development in Section 4.2, $C^{\prime}$ for an equilateral triangle can be expressed as

$$
C^{\prime}=2\left[\frac{2(n+2)(n+3)}{\sqrt{3}}\right]^{1 / n}
$$

Thus, for an equilateral triangular beam, $C_{E}$ equals to $4 / 3$, and for $n \approx 0.26, C$ has a value of 0.716 . 


\section{A.2. Square cross-section}

For a square cross-section, $C_{I}=1 / 12$ and

$$
C_{f 0}=\frac{8 \sqrt{3}}{9\left(1-\cos \phi_{0}\right)\left(\sin \phi_{0}\right)^{2}}
$$

$$
C^{\prime}=2(n+2)^{1 / n}
$$

Thus for the square cross-section with $\phi_{0}=\alpha, C_{E}$ is 1.15 and $C$ is 0.689 for $n \approx 0.26$; these values are slightly less than those for the equilateral triangular section. 